\title{
Calculation Method for Optimal Routing in Traffic Network Big Data
}

\author{
Sun Hongfeng ${ }^{*}$, Li Ying and Yang Yanchun \\ Information Technology School, Shandong Women's University, Jinan, 250030, China
}

\begin{abstract}
In allusion to the influence of traffic flow change on vehicle routing selection, the article aims at finding the method for planning the trip route under the condition of knowing traffic flow change. The method combining particle swarm algorithm and dynamic planning is used for routing optimization in order to obtain the excellent route for various vehicles influenced by traffic flow. The road network structure and the traffic data in actual environment are adopted for simulation and the result shows that this method can truly and dynamically optimize routing, and meanwhile the road traffic flow and the travel time of vehicles can also significantly influence the routing selection of vehicles.
\end{abstract}

Keywords: Dynamic planning, particle swarm algorithm, routing optimization, time dependence, traffic flow.

\section{INTRODUCTION}

The vehicle routing planning based on traffic flow makes road network diversified and true. Routing optimization not only aims at optimizing the service sequence among client points, but also aims at optimizing the routing among the client points, with the optimization objective converted from shortest distance to shortest time. In modern society, time gradually becomes an important asset in people's daily life and accordingly time use efficiency becomes the essential condition for the benefit maximization in various industries. For logistics industry, along with the increased logistics cost, how to timely and efficiently complete transportation tasks becomes logistics enterprises' problem that shall be urgently solved. With the features of less individuals, simple operation, fast convergence rate, easy realization, etc., particle swarm algorithm has been widely applied by the scholars in the field of computer science and management science and meanwhile has obtained a lot of research achievements. $\mathrm{Wu}$ Kaijun, et al., have adopted the binary coded particle swarm algorithm for vehicle routing optimization [1-4]. Zhang Liyan and Zhang Wenjing both have proposed the application of particle swarm algorithm in vehicle routing problem [5, 6]. Self-adaptively weighted particle swarm algorithm has been adopted in the article to initially optimize client points and vehicles and then optimize the routing among the client points by stages according to road traffic flow change and finally obtain the optimal distribution routes of various vehicles.

\section{CALCULATION METHOD FOR OPTIMAL ROUT- ING IN TRAFFIC NETWORK BIG DATA}

The vehicles researched in the article refer to $\mathrm{m}$ vehicles starting from the distribution center and orderly distributing cargoes to client points. If client points cannot timely reached as scheduled, then time penalty is adopted.

\section{Objective function}

$$
\begin{gathered}
\min z=\sum_{m \in M} \sum_{k \in V_{m}}\left\{r \cdot\left[d\left(m, x_{1}\right)+\sum_{x_{j} \in C_{m}^{k}} d\left(x_{j-1}, x_{j}\right)+d\left(x_{\mid C_{m}}^{k}, m\right)\right]+\right. \\
{\left[\sum_{x_{j} \in C_{m}^{k}}\left[f \cdot \max \left(s t_{x_{i}}-t_{x_{i}}^{k}, 0\right)+f_{x_{i}} \cdot \max \left(t_{x_{i}}^{k}-e t_{x_{i}}, 0\right)\right]\right\}+} \\
\sum_{m, n \in M}\left[2 r \cdot d(m, n) \cdot \max \left(\left[\sum_{c \in C} y_{c m n} g_{c} / \omega\right],\left[\sum_{c \in C} y_{c m n} g_{c} / \omega\right]\right)\right]
\end{gathered}
$$

Constraint condition

$$
\begin{aligned}
& \text { st. } V_{p} \cap V_{q}=\varnothing\left(\left|V_{p} \cap V\right|_{q}=0\right) \forall p, q \in M \\
& C_{1}^{1} \cup C_{1}^{2} \cup \cdots \cup C_{1}^{\left|V_{1}\right|} \cup \cdots \cup C_{|M|}^{1} \cup C_{|M|}^{2} \cup \cdots \cup C_{|M|}^{\left|V_{|M|}\right|}=C \\
& C_{p}^{u} \cap C_{p}^{v}=: \varnothing \quad \forall p, q \in M \quad \forall u \in V_{p}, \forall v \in V_{q} \\
& y_{x_{i} \operatorname{center}\left(x_{i}\right) m}=\left\{\begin{array}{l}
1, m \neq \operatorname{center}\left(x_{i}\right) \\
0, \text { others }
\end{array} \forall m \in M \quad k \in V_{m}, \forall x_{i} \in C_{m}^{k}\right. \\
& \operatorname{Min} Z=F_{\text {time }} \sum_{a \in s} \sum_{b \in s} \sum_{t=\alpha}^{\beta-1} x_{a b}^{t} T_{a b}^{t}+F_{w} \sum_{a \in s} w_{a}+F_{d} \sum_{a \in s} d_{a}
\end{aligned}
$$

In above formulae, the first part is the total time cost of vehicle, the second part denotes the total travel distance cost of vehicle, the third part denotes the penalty cost generated due to the unpunctual service of vehicles, wherein $S$ denotes client points set, $p$ denotes client points $\&$ road nodes set, $w_{a}$ denotes the waiting time of client point $a, d_{a}$ denotes the delay time of client point $a, F_{\text {time }}$ denotes the travel cost in unit time, $F_{w}$ denotes the waiting cost coefficient of vehicle, 
$F_{d}$ denotes the delay cost coefficient of vehicle, $T_{a b}^{t}$ denotes the time for the vehicle to travel from client point $a$ at time $\mathrm{t}$ to client point $b$ and $x_{a b}^{t}$ is decision variable (if the vehicle starts from client point $a$ at time to client point $b$, then $x_{a b}^{t}=1$; or else, $x_{a b}^{t}=0$ ).

The constraint condition of the problem is as follows:

1) Constraint for the departure time $t_{a}$ and the arrival time $t_{a}^{e}$ of vehicle at client point $a$ : $\left[t_{s}, t_{e}\right]$ denotes the time window of the planning period.

$$
t_{a}^{e}=t_{a}+T_{a j}^{t} t_{a} \in\left(t_{s}, t_{e}\right], t_{a}<t_{a}^{e} \leq \beta, a \in S, j \in P
$$

2) Calculation of the waiting time and the delay time of client point $a$ : $p_{a}$ denotes the expected service time of client point $a$ and $e_{a}$ denotes the arrival time at client point $a$.

$$
\begin{aligned}
& w_{a}=\operatorname{Max}\left(0, p_{a}-e_{a}\right), a \in S \\
& d_{a}=\operatorname{Max}\left(0, e_{a}-p_{a}\right), a \in S
\end{aligned}
$$

3) Constraint for client point to be visited by one vehicle

$$
\sum_{\substack{t=a \\
j \in P \\
j \neq a}}^{w} x_{j a}^{t}-\sum_{\substack { t=a \\
\begin{subarray}{c}{i \in P \\
a \neq i{ t = a \\
\begin{subarray} { c } { i \in P \\
a \neq i } }\end{subarray}}^{w} x_{a i}^{t}=0 \quad a \in S
$$

4) Capacity constraint: $q_{a}$ denotes the quantity of the cargoes needed by client point $a, Q$ denotes the rated loading capacity of vehicle, $y_{a}^{k}$ is a decision variable (if vehicle k passes by client point $a$, then $y_{a}^{k}=1$; or else, $y_{a}^{k}=0$ ), $\sum_{a \in s} q_{a} y_{a}^{k} \leq Q$

When calculating the time for travelling among the client points, it is necessary to consider time, road segment and other relevant factors. The departure time at the client point shall be determined according to the departure time $t_{a}$ of the vehicle before entering the client point and the traveling time $T_{a b}^{t}$ thereof. For the vehicle routing among the client points or from the distribution center to the client points, two-end rolling searching method can be adopted to determine the road segments included in the route and the specific steps are as follows:

The $1^{\text {st }}$ step: search all nodes that are connected to client point $a$ (distribution center) through arc lines and use $v_{a}$ to stand for the nodes set.

The $2^{\text {nd }}$ step: search all nodes that are connected to client point $b$ (distribution center) through arc lines and use $v_{b}$ to stand for the nodes set.

The $3^{\text {rd }}$ step: compare $v_{a}$ and $v_{b}$ to find the common points; if there is any common point, include the route between client point $a$ and client point $b$ in route set R; if there is no common point, then continue to search around the point in $v_{a}$ or $v_{b}$ on the principle of minimizing the number of passed nodes and meanwhile compare the searched point with $v_{a}$ or $v_{b}$; set the number of nodes as $\varepsilon$, and stop the searching operation when this value $\varepsilon$ is exceeded.

Obtain the route between client $a$ and client $b$ according to above calculation, and find the road segment $E_{i j}$ in the road network regarding this route, wherein $i$ and $j$ refer to the road segment connection nodes in the road network. $t_{i j}$ denotes the time of entering road segment $E_{i j}$ and $q_{t_{i j}}$ denotes the traffic flow of road segment at time $t_{i j}$, and then the above formulae (4) and (5) can be used to solve the average velocity $v_{t_{i j}}$ for the vehicle to pass through road segment $E_{i j}$. On the basis of referring to the travel time calculation by virtue of such methods as queuing theory and intersection delay mentioned in Cao Xiangyu's paper and meanwhile neglecting the influence of intersection delay on travel time for calculation simplification [7-10], the following travel time calculation formula for road segment $E_{i j}$ is obtained:

$$
\begin{aligned}
& T_{t_{i j}}=\frac{L_{i j}}{v_{t_{i j}}} \\
& v_{t_{i j}}=\frac{a_{1} v_{0}}{1+\left(q_{t_{i j}} / c\right)^{\beta}}
\end{aligned}
$$

In the formula, $L_{i j}$ denotes the length of road segment $E_{i j}$, same $v_{0}$ and $c$ are assumed for each road segment. Meanwhile, it is only necessary to consider the traffic flow change along with the change of time and road position and then calculate the time cost according to the obtained travel time and finally find the route with lowest time cost.

\section{ALGORITHM DESCRIPTIONS}

\subsection{Particle Encoding Mode in Vehicle Routing Problem}

Vehicle routing problem is an integer programming problem, but the searching space of particle swarm is continuous, so how to make each particle correspond to the problem solution is the key point for algorithm implementation. On the basis of referring to literature, a VRP problem with M task points corresponding to a $2 \mathrm{M}$-dimensional space is established in the article, and the $2 \mathrm{M}$-dimensional vector $X$ corresponding to each particle is divided into two M-dimensional vector: $X_{i v}$ (denotes the vehicle corresponding to each task) and $X_{i r}$ (denotes the priority sequence in the vehicle routing corresponding to each task).

For example, for a vehicle routing problem including 7 client points and 3 vehicles, the position vector $X$ of the i-th particle is:

Round off $X_{i v}$ to obtain1, 2, 2, 3, 3, 1, 3; use $X_{i r}$ to arrange the client sequence so as to obtain the task allocation of each vehicle and the sequence for serving the client $(0$ denotes the distribution center) 
Table 1. Particle vector.

\begin{tabular}{|c|c|c|c|c|c|c|c|}
\hline Client Point & $\mathbf{1}$ & $\mathbf{2}$ & $\mathbf{3}$ & $\mathbf{4}$ & $\mathbf{5}$ & $\mathbf{6}$ & $\mathbf{7}$ \\
\hline \hline$X_{i v}$ & 1.7 & 2.3 & 2.6 & 3.4 & 3.9 & 1.2 & 3.6 \\
\hline$X_{i r}$ & 0.9 & 2.8 & 3.7 & 1.4 & 2.6 & 4.4 & 1.8 \\
\hline
\end{tabular}

Table 2. Client points data.

\begin{tabular}{|c|c|c|c|}
\hline Client Point $\mathbf{i}$ & $q_{i}$ & $p_{i}$ & Coordinate \\
\hline \hline 1 & 2 & 33 & {$[40,21]$} \\
\hline 2 & 3 & 60 & {$[24,43]$} \\
\hline 3 & 3.5 & 110 & {$[35,67]$} \\
\hline 4 & 2.5 & 72 & {$[60,48]$} \\
\hline 5 & 4 & 119 & {$[78,50]$} \\
\hline 6 & 1.5 & 44 & {$[60,30]$} \\
\hline 7 & 3 & 123 & {$[83,70]$} \\
\hline 8 & 4.5 & 73 & {$[80,35]$} \\
\hline
\end{tabular}

Vehicle 1: 0-1-6-0

Vehicle 2: 0-2-3-0

Vehicle 3: 0-4-7-5-0

\subsection{Algorithm Procedure Description}

The $1^{\text {st }}$ step: algorithm initialization: input the corresponding parameters of road network, determine particle swarm scale $n$, inertia factor $\omega$, learning factors $c_{1}$ and $c_{2}$, maximum iteration times $N_{\max }$.

The $2^{\text {nd }}$ step: particle swarm initialization: randomly assign the real number in the interval of $1 \sim K$ (number of vehicles) for each particle vector $X_{i v}$, and randomly assign the real number in the interval of $1 \sim L$ (number of tasks) for each dimension of $X_{i r}$; randomly assign the real number in the interval of $-(K-1) \sim(K-1)$ (number of vehicles) for each dimension of each velocity vector $V_{i v}$ and randomly assign the real number in the interval of $-(L-1) \sim(L-1)$ for each dimension of $V_{i r}$, wherein the boundary value of the corresponding value range is namely the boundary value for particle activity.

The $3^{\text {rd }}$ step: fitness assessment: form vehicle distribution scheme for particle decoding, use two-end rolling searching method to determine the routing among client points according to the positions of the client points, and then calculate the travel time of each routing plan according to time dependence and travel distance so as to accordingly calculate the route and the travel time of each distribution scheme, and finally calculate the fitness of the objective function and meanwhile check whether the scheme can meet the con- straint conditions (1) (4); if the constraint conditions cannot be met, search route again [11].

The $4^{\text {th }}$ step: for each particle, compare the fitness and the best position pbest of the particle and select individual optimal extremum; for each particle, compare the fitness and the best position pbest of all particles and select global optimal extremum; update the individual extremum and the global extremum of particle swarm.

The $5^{\text {th }}$ step: according to formulae (7) and (8), update the velocity and the position of each particle in the particle swarm.

The $6^{\text {th }}$ step: judge whether the present iteration times reach the preset maximum times; if yes, stop iteration and output the optimal solution; or else, forward to the $3^{\text {rd }}$ step [12].

\section{SIMULATION EXPERIMENT}

\subsection{Experiment Description and Parameter Setting}

Experiment environment: lenovo corei5, 2G memory, WindowsXP, MATLAB17.0.

For the experiment in the article, the road network including 105 nodes and 164 road segments is taken according to the road network condition in a certain city and the simulation tool Netlogo is used to simulate the traffic flow change of each urban road segment on the basis of the actual operating condition of the vehicles in the road network in order to obtain the basis data value of the traffic flow of each road segment. Then MATLAB is used to test the obtained basic data. This experiment is carried out for the distribution optimization involving in 8 client points and 3 vehicles, wherein the maximum loading capacity of each vehicle is $8 \mathrm{t}$ and the client point data are as shown in Table $\mathbf{1}$ [13]. 
All vehicles start from the distribution center, and the velocity of each vehicle shall be determined according to the departure time and the velocity change determined according to Table 2 for the vehicle at the road segment. Therein, the penalty coefficient for unpunctual arrival at the client point is $F_{w}=F_{d}=10$, unit travel time $\operatorname{cost} F_{\text {time }}=5$ and number of nodes $\varepsilon=7$. The parameters of particle swarm algorithm include: particle swarm scale $n=50$, particle dimensions $D=16$, iteration times $N_{\max }=50$ and experiment repetition times $=10$.

\subsection{Experiment Result}

Route and relevant parameters at time $t_{1}$ (the part in bracket denotes the time of arriving at the client point or passing by the road segment):

In the calculation example, one the one hand, vehicle travel time is compared under the condition of changed time but constant route; on the other hand, the influences of different starting routes on travel time are compared under the condition of the same departure time [14]. Such controlled comparison method can improve the comparability and readability of the algorithm result.

According to various experiment, the influence of traffic flow on vehicle routing planning is mainly presented in the following aspects: for the vehicles have different departure time but same transport destination, the vehicle routings are different from each other and such routing difference can be obviously found in the experiments; and meanwhile the vehicles starting at different time also have different client point arrival accuracies. In actual transport environment, the traffic flow can significantly influence vehicle routing selection and planning [15].

\section{CONCLUSION}

The road network chart based on traffic flow information is proposed in the article for routing planning. After introducing the traffic flow, it is necessary to consider the influence of the traffic flow on average velocity of the vehicles on the road segment, wherein the traffic flow is constantly changed in the whole day. The article includes two innovation points: firstly, the influence of the traffic flow on routing selection and planning is considered during routing planning, thus to be different from the vehicle routing planning in static environment; secondly, the routing selection among client points is importantly considered during the service optimization of client points. The client point optimization includes the following two aspects: firstly, optimization of client sequence; secondly, optimization of routing selection among client points. In the article, particle swarm algorithm is adopted for the planning of client point service sequence and the dynamic planning method is adopted for the planning of the routing among the client points. The experiments show that the routing planning based on traffic flow change can reduce transport time and improve cargo distribution timeliness as well as reduce transport cost.

\section{CONFLICT OF INTEREST}

The authors confirm that this article content has no conflict of interest.

\section{ACKNOWLEDGEMENTS}

This paper is supported by Natural Science Foundation of Shandong Province, No. ZR2013FL026 and University Science and Technology Projects in Shandong Province, No.J14LN01.

\section{REFERENCES}

[1] J. He, Y. Geng and K. Pahlavan, "Modeling Indoor TOA Ranging Error for Body Mounted Sensors," 2012 IEEE 23nd International Symposium on Personal Indoor and Mobile Radio Communications (PIMRC), Sydney, Australia, pp. 682-686, 2012.

[2] Y. Geng, J. Chen, K. Pahlavan, "Motion detection using RF signals for the first responder in emergency operations: A PHASER project[C]," 2013 IEEE 24nd International Symposium on Personal Indoor and Mobile Radio Communications (PIMRC), London,Britain, 2013.

[3] S. Li, Y. Geng, and J. He, "K. Pahlavan,Analysis of Threedimensional Maximum Likelihood Algorithm for Capsule Endoscopy Localization," 2012 5th International Conference on Biomedical Engineering and Informatics (BMEI), Chongqing, China, pp. 721-725, 2012.

[4] Y. Geng, J. He, H. Deng and K. Pahlavan, "Modeling the Effect of Human Body on TOA Ranging for Indoor Human Tracking with Wrist Mounted Sensor," 16th International Symposium on Wireless Personal Multimedia Communications (WPMC), Atlantic City, NJ, 2013.

[5] Y. Geng, J. He, And K. Pahlavan, "Modeling The Effect Of Human Body On Toa Based Indoor Human Tracking," International Journal of Wireless Information Networks, vol. 20, no. 4, pp. 306-317, 2013.

[6] Z. Lv, T. Yin, Y. Han, Y. Chen, and G. Chen, "WebVR--Web Virtual Reality Engine Based on P2P network," Journal of Networks, vol. 6 , no. 7, 2011.

[7] Z. Lv, "Wearable smartphone: Wearable hybrid framework for hand and foot gesture interaction on smartphone." In Computer Vision Workshops (ICCVW), In:International Conference on, pp. 436-443, 2013.

[8] Z. Lv, L. Feng, H. Li, and S. Feng, "Hand-free motion interaction on Google Glass," In SIGGRAPH Asia 2014 Mobile Graphics and Interactive Applications, pp. 21, 2014.

[9] C. Zhong, S. M. Arisona, X. Huang, M. Batty, and G. Schmitt, "Detecting the dynamics of urban structure through spatial network analysis," International Journal of Geographical Information Science, vol. 28, no. 11, pp. 2178-2199, 2014.

[10] W. Li, J. Tordsson, and E. Elmroth, "An aspect-oriented approach to consistency-preserving caching and compression of web service response messages," In: Web Services (ICWS), International Conference on, pp. 526-533, 2010.

[11] Z. Lv, and T. Su, "3D seabed modeling and visualization on ubiquitous context," In: SIGGRAPH Asia 2014 Posters, p. 33. ACM, 2014.

[12] Z. Lu, L. Feng, S. Feng, and H. Li,. "Extending Touch-less Interaction on Vision Based Wearable Device," Virtual Reality (VR), 2015 .

[13] M. Zhang, Z. Lv, X. Zhang, G. Chen, and K. Zhang, "Research and Application of the 3D Virtual Community Based on WEBVR and RIA," Computer and Information Science, vol. 2, no. 1, pp. 84, 2009. 
[14] T. Su, Z. Lv, S. Gao, X. Li, and H. Lv, "3D seabed: 3D modeling and visualization platform for the seabed," In: Multimedia and Expo Workshops (ICMEW), International Conference on, pp. 1-6, 2014.
[15] D. Jiang, Z. Xu, P. Zhang, and T. Zhu, "A transform domain-based anomaly detection approach to network-wide traffic." Journal of Network and Computer Applications, vol. 40, pp. 292-306, 2014.

Received: June 10, 2015

Revised: July 29, 2015

Accepted: August 15, 2015

(C) Hongfeng et al.; Licensee Bentham Open.

This is an open access article licensed under the terms of the (https://creativecommons.org/licenses/by/4.0/legalcode), which permits unrestricted, noncommercial use, distribution and reproduction in any medium, provided the work is properly cited. 